\title{
Social Processes, Program Verification and All That
}

\author{
ANDREA ASPERTI, ${ }^{1}$ HERMAN GEUVERS ${ }^{2}$ and RAJA NATARAJAN ${ }^{3}$ \\ ${ }^{1}$ Dept. of Comp. Sci., Univ. of Bologna, Mura Anteo Zamboni 7, 40127 Bologna, Italy \\ Email: asperti@cs.unibo.it \\ ${ }^{2}$ Dept. of Comp. Sci., Radboud Univ. Nijmegen and Tech. Univ. Eindhoven, The Netherlands \\ Email: herman@cs.ru.nl \\ ${ }^{3}$ School of Tech. $\&$ Comp. Sci., Tata Institute of Fundamental Research, Mumbai 400 005, India \\ Email: raja@tifr.res.in
}

Received 25 April 2009; Revised 3 May 2009

\begin{abstract}
In a controversial paper at the end of 1970's, R.A. De Millo, R.J. Lipton and A.J. Perlis (DeMillo, Lipton and Perlis, 1979) argued against formal verifications of programs, mostly motivating their position by an analogy with proofs in mathematics, and in particular with the impracticality of a strictly formalist approach to this discipline. The recent, impressive achievements in the field of interactive theorem proving provide an interesting ground for a critical revisiting of those theses. We believe that the social nature of proof and program development is uncontroversial and ineluctable but formal verification is not antithetical to it. Formal verification should strive not only to cope, but to ease and enhance the collaborative, organic nature of this process, eventually helping to master the growing complexity of scientific knowledge.
\end{abstract}

\section{Introduction}

Heavier than air flying machines are impossible.

Formal verification of programs, no matter how obtained, will not play the same role in the development of computer science and software engineering as proofs do in mathematics.

- R.A. De Millo, R.J. Lipton, A.J. Perlis (DeMillo et al., 1979)

Samuel Pierpont Langley was a professor of astronomy and physics, and a world-expert in aerodynamics during the late nineteenth and early twentieth century. The esteem with which he is held can be seen from the fact that one of the research centers of NASA is named after Langley. At the height of his research career, Samuel Langley published a result (Langley, 1891) which came to be known as "Langley's Law." According to this erroneous law - higher the speed, lower the drag - more power was required in order to make an aircraft fly slower, and if this were to be true then heavier than air flying machines would certainly have been an impossibility. Fortunately the Wright Brothers 
had not read Langley's book, and they went on to develop the first manned aircrafts which could be controlled in-flight from the aircraft itself.

In a famous, influential paper at the end of 1970's, R.A. De Millo, R.J. Lipton and A.J. Perlis (DeMillo et al., 1979) advanced various criticisms about the very idea of formal verifications of programs. The impressive advances in this area seem by themselves to belie their gloomy predictions. Formal verification has currently reached such a level of maturity, as to allow correctness proofs of sophisticated hardware components (Harrison, 2007), complex programs such as optimizing compilers (see e.g.(Leroy, 2006; Tristan and Leroy, 2008)), and also parts of modern operating systems (see e.g. (?; Klein, 2009)).

It is precisely in view of these achievements, however, that we can look back at (DeMillo et al., 1979) with a less passionate and more objective spirit, making a more stringent analysis of their thesis and arguments, without focusing on the polemic frame intentionally chosen by its authors, viz., in Lamport's words ${ }^{\dagger}$, as a debate between a reasonable engineering approach that completely ignores verification and a completely unrealistic view of verification advocated only by its most naive proponents.

In fact, some of the thesis advocated by De Millo, Lipton and Perlis are sharable, very pertinent and still relevant; on the other hand, most of their arguments, after thirty years of research, sounds obsolete and a bit trite, asking for a critical reappraisal. Here is a quick summary of our critique, before we enunciate it in detail.

- De Millo et al. state that ... intuition is the final authority (quoting the logician Rosser).: Intuitions and analogies may help in the explanation and the assimilation of a statement, but when it comes to verification of a statement, proof is the authority. Intuition sometimes just fails.

— De Millo et al. state that ... we will continue to argue that programming is like mathematics, and that the same social processes that work in mathematical proofs doom verifications.: We argue that mathematics will become more and more like programming and that the future of both mathematics and programming lies in the fruitful combination of formal verification and the usual social processes that are already working in both scientific disciplines.

\section{Proofs and programs}

Russell did succeed in showing that ordinary working proofs can be reduced to formal, symbolic deductions. But he failed, in three enormous, taxing volumes, to get beyond the elementary facts of arithmetic. He showed what can be done in principle and what cannot be done in practice.

- R.A. De Millo, R.J. Lipton, A.J. Perlis (DeMillo et al., 1979)

In making a parallel between program verification and proofs of theorems the key arguments adduced by De Millo et al. against the formal approach is the essential impracticality of a strictly logistic approach to mathematics, due to the nearly inconceivable length of a deduction from first principles. The argument is repeated several times: The quotation above is from their paper (page 272). And again, in the next page,

\footnotetext{
$\dagger$ http://research.microsoft.com/en-us/um/people/lamport
} 
A formal demonstration of one of Ramanujan's conjectures assuming set theory and elementary analysis would take about two thousand pages.

At page 275, Poincaré is quoted in support of their claim:

If it requires twenty-seven equations to establish that 1 is a number, how many will it require to demonstrate a real theorem?

Of course, the argument is not new; even Bourbaki, who is traditionally enlisted in the ranks of the formalist school (Lee, 2002), found the project of formalizing mathematics absolutely un-realizable (Bourbaki, 1968):

the tiniest proof at the beginning of the Theory of Sets would already require several hundreds of signs for its complete formalization.

The argument is reminiscent of the general disbelief about the potentialities of computers, and the possibilities of writing long programs at the beginning of the fifties, just due to the inability to conceive high-level languages and a process of automatic translation to a machine-understandable code. When Grace Hopper wrote the first compiler in 1952, opening the way to software development, nobody looked interested: "I had a running compiler and nobody would touch it", she said. "They told me computers could only do arithmetic." (Schieber, March/April 1987). The analogy with compilation was already made by Maurer, back in 1979 (Maurer, 1979):

We can make an analogy here with compiling a higher level language program into a machine language. Originally this was done by hand [...], then compilers came along and started to do the job automatically. [...] nobody is ever going to read the object code produced by a compiler; one simply trusts the compiler. What we hope for in verifiers is that we will at least be able to trust them to show program correctness.

Harrison (Harrison, 2008) has recently restated the concept in the following terms:

the arrival of the computer changes the situation dramatically. [...] checking conformance to formal rules is one of the things computers are very good at. [...] the Bourbaki claim that the transition to a completely formal text is routine seems almost an open invitation to give the task to computers.

In fact, automation of formal reasoning has recently gone far beyond the elementary facts of arithmetic, permitting the formalization and automatic verification of complex results such as the asymptotic distribution of prime numbers (Avigad, Donnelly, Gray and Raff, 2007), the four color theorem (Gonthier, December 15-17, 2007; Gonthier, 2008) or the Jordan curve theorem (Hales, 2007). All these developments are conspicuous (spanning from 30 to 75 thousand lines of code), but their complexity is still negligible when compared with, say, the size of a modern operating system.

The formal proof of the Jordan curve theorem is due to Thomas Hales, a famous mathematician, particularly known for his proof of the Kepler conjecture (the most compact way of packing congruent spheres in three dimensional Euclidean space), and for the events related to its publication. Briefly, his proof of the Kepler conjecture involved large amounts of computer verification, and after three years of work, the reviewers of the Annals of Mathematics concluded that, although they believed the proof was correct, they were unable to check it thoroughly, due to many "low-level components" that lacked a more general intuition, especially given the degree of computation involved. At the end, 
the Annals of Mathematics published a short version of the proof (Hales, 2005), and made the code/data for the proof available un-reviewed on its website. A revised, full version of the article was finally published by Discrete and Computational Geometry. Since then, Thomas Hales started a new project, called Flyspeck (see (Hales, 2008)), to formally check the correctness of his proof with the help of interactive theorem provers.

The Kepler conjecture and the four color theorem are good examples of a large number of mathematical proofs based on a direct and substantial use of the computer. Other examples mentioned by Hales in (Hales, 2008) are the non-existence of a projective plane of order 10, the proof that the Lorentz equations have a strange attractor, the double-bubble problem for minimizing soap bubbles enclosing two equal volumes, the optimality of the Leech lattice among 24-dimensional lattice packings, and hyperbolic 3-manifolds. In all these cases, the computer is used to manage the complexity, usually by automatically checking a finite, albeit conspicuous number of "atomic" configurations (a kind of computation that would not be possible, or extremely laborious for a human). Mathematics is entering a new era of results requiring proofs of a complexity and dimension that defy human comprehension, leaving a miasma of doubt about their effective correctness. For instance, the theorem of classification of finite groups is the result of the collective work of about a hundred authors, composed of over 10000 pages of results, spread across 500 journal articles. One of the key results, the Feit-Thomson (or odd-order) theorem (Feit and Thompson, 1963) takes 255 pages to itself. A formalization of the odd-order theorem has recently been started by the INRIA-Microsoft research project on "mathematical components" lead by G. Gonthier (Gonthier, Mahboubi, Rideau, Tassi and Thery, 2007). As another example, the preprint of F. Almgren's masterpiece in geometric measure theory, familiarly referred to as the "Big Paper", is 1728 pages long.

Maybe for the first time in the history of their discipline, mathematicians are forced to accept the simple fact that many theorems, even admitting simple and elegant statements, may not admit equally simple and elegant proofs ${ }^{\ddagger}$. As observed in (Geuvers, 2009), it can be formally proved that, in any given logical system, there is no upper bound to the fraction between the size of a statement and the size of its shortest proof, and there is no reason to believe that things should be better when restricted to "interesting" theorems. Even De Millo et al. themselves admit that this is the case.

For even the most trivial mathematical theories, there are simple statements whose formal demonstrations would be impossibly long.

But they don't want to accept the consequence that a computer may be needed to help the human in verifying the formal demonstrations.

Not always can one find "a truly marvelous demonstration" that, alas, is just a bit too long to "fit in the margin of a book": a proof can just be so complicated, no matter what kind of rethinking you might try. Does this mean that such a proof has just to be dismissed and the validity of the statement rejected, possibly renouncing any proof, all sacrificed on the mystic altar of elegance and simplicity? Doesn't it possibly mean that

$¥$ According to Lakatos (Lakatos, 1976), simplicity is the eighteenth-century idea of mathematical rigor. 
Table 1. The verifier's analogy, according to De Millo, Lipton and Perlis

\begin{tabular}{ll}
\hline \hline Mathematics & Programming \\
\hline theorem & program \\
proof & verification \\
\hline \hline
\end{tabular}

Table 2. De Millo, Lipton and Perlis' analogy

\begin{tabular}{ll}
\hline \hline Mathematics & Programming \\
\hline theorem & specification \\
proof & program \\
imaginary formal proof & verification \\
\hline \hline
\end{tabular}

we merely have to look for the right tools aiding us to cope with its complexity, and that we have to learn to appreciate a different, less archaic, kind of beauty?

\section{Theorems, Proofs and Specifications}

By far the most common way in which we deal with something new is by trying to relate the novelty to what is familiar from past experience: we think in terms of analogies and metaphors. (Even the 5th Edition of the Concise Oxford Dictionary still defines a typewriter as a "machine for printing characters on paper as substitute for handwriting"!) As long as history evolves along smooth lines, we get away with that technique, but that technique breaks down whenever we are suddenly faced with something so radically different from what we have experienced before that all analogies, being intrinsically too shallow, are more confusing than helpful.

\section{- EDsGer DiJkstra (Edsger.W.Dijkstra, 1986)}

De Millo, Lipton and Perlis describe the "verifier's analogy" between mathematics and programming (see Table 1), and they contrast it with their own analogy (Table 2). Their observation is that the verifiers are mistaken by wanting to identify the notion of "proof" (from mathematics) with "formal verification" in computer science.

De Millo, Lipton and Perlis do not give any source for the analogy attributed to "verifier's"; in fact, it can be hardly imagined that anybody working in the area of program verification would feel at ease with such an analogy. If somebody working on formal methods really put it forward, their intent was probably to emphasize a simple but crucial fact, which, at the end of seventies was still hard to grasp, namely that programs themselves could become, like mathematical theorems, the object of a scientific investigation.

In fact, the analogy that De Millo, Lipton and Perlis are so happy with, to the extent to believe they invented it themselves, was precisely the leading theme that, in a slightly more sophisticated form, was right at that time driving research in the field of computer assisted verification. The "formal" counterpart of the analogy is called Curry-Howard correspondence (Howard, 1980), and it merely differs from that in Table 2 by the fact 
Table 3. Curry-Howard Correspondence

\begin{tabular}{ll}
\hline \hline Mathematics & Programming \\
\hline theorem & type \\
proof & program \\
correctness verification & type checking \\
cut elimination & computation \\
\hline \hline
\end{tabular}

\section{Cut Elimination}

A cut is a particular logical rule that permits the factorization of a complex reasoning step into a sequence of simpler steps. In order to prove $A$, we may temporarily assume $B$, provided we prove it later. In principle, one expects to be able to entirely avoid the use of this rule, by simply unfolding the proof of $B$ inside the original proof of $A$. In practice, the cut-elimination proof, also known as Gentzen's Hauptsatz, is far from trivial, and is not satisfied by all logical systems; but when it holds, it is rich in consequences:

Consistency: It is usually easy to verify that a system does not admit cut-free proofs of the absurd. In such a case, if the system enjoys the cut elimination property, it is immediately consistent.

Subformula property: This is an important property in several approaches to prooftheoretic semantics and automated theorem proving. In essence, it says that in order to prove a given statement $A$, you only need to use sub-formulae of $A$.

Fig. 1. Normalization of Proofs

of substituting "type" for "specification": a simple twist, opening by itself an entire new universe of possibilities ${ }^{\S}$. The analogy can then be made more precise (see Table 3). Proof verification, is nothing other than type-checking, and also computation has a prooftheoretical counterpart in the form of cut-elimination, that is a process of normalization of proofs essentially consisting in removing "detours" (lemmas) by inlining them (see Fig. 1).

The analogy with type checking also helps in clarifying a common misconception about automatic verification. When we type-check a piece of code, a mathematical expression, say, we do not have to compute it in order to check that it is properly typed: type checking is an entirely static operation. Similarly, when we check the proof of a mathematical theorem - the famous Ramanujan's conjecture, say - we are not supposed to first normalize the proof to a mere application of axioms from set theory and elementary analysis: the proof can make use of any sort of theorems and lemmas, and we check it statically and compositionally.

Starting from their analogy (see Table 2), De Millo, Lipton and Perlis also raise the following fundamental logical objection to verification:

Since the requirement for a program is informal and the program is formal, there must be a transition, and the transition itself must necessarily be informal.

$\S$ Howard's paper was printed in 1980, but the first draft circulated in 1969. As observed by Howard himself, however, the main ideas are to be ascribed to Curry, back in the fifties. 
The criticism was already answered by Maurer (Maurer, 1979), distinguishing between program correctness (the fact that a program meets a specification), and specification correctness (the fact that the specification meets the user expectations):

A proof of correctness consists of two steps, one formal, the other informal; and neither of the two is valid without the other one.

The idea of the Curry-Howard correspondence is that not only specifications can be given in a completely formal way, but that, by suitably enriching the system of types, and in particular by adding dependent types, they can be altogether assimilated to types. A dependent type is a type which depends on a term. A typical example is the type of $n$-dimensional vectors is some space $\mathcal{A}$, whose type clearly depends on the value $n$. More generally, given a specification $R(x, y)$ expressing the expected relation between the input $x: A$ and the output $y: B$ of a program (where $A$ and $B$ can be seen as "traditional" types), we can build the following dependent type $\forall x: A .\{y: B \mid R(x, y)\}$ and try to check if our program inhabits it. Of course, in order to perform the verification the programmer may be forced to provide substantial help to the type-checker in the form of suitable type annotations of inner expressions (not too far from pre and post conditions in an axiomatic setting (Hoare, 1969)). Quoting Altenkirch et al. (Altenkirch, McBride and McKinna, n.d.):

While conventional type systems allow us to validate our programs with respect to a fixed set of criteria, dependent types are much more flexible, they realize a continuum of precision from the basic assertions we are used to expect from types up to a complete specification of the program's behavior. [...] While the price for formally certified software may be high, it is good to know that we may pay it in instalments and that we are free to decide how far we want to go. Dependent types reduce certification to type checking, hence they provide a means to convince others that the assertions we make about our programs are correct. Dependently typed programs are, by their nature, proof carrying code (Necula and Lee, 1996; Hamid, Shao, Trifonov, Monnier and $N i$, 2003).

The Curry-Howard correspondence also opens up a completely innovative perspective on program verification, not consisting in trying to match a program against a specification, but merely consisting in proving that the specification can be inhabited. If the user can supply a constructive proof of this fact, then it is possible to automatically extract from the proof its algorithmic content, i.e. a program satisfying the specification. Program extraction was exploited for the first time in the Nuprl proof development system (Constable, Allen, Bromley, Cleaveland, Cremer, Harper, Howe, Knoblock, Mendler, Panangaden, Sasaki and Smith, 1986). Having proved a property $t$ of the form $\forall x: A, \exists y: B, R(x, y)$, the term term_of ( $\mathrm{t}$ ) extracts a function mapping any $a$ of type $A$ into a pair consisting of an element $b$ of type $B$ and a proof $p$ that such a $b$ verifies the property $R(a, b)$. By selecting the first component of this pair, we have a function $f$ from $A$ to $B$ such that $R(x, f(x))$ for all $x$ in $A$. The extraction technique can also be extended to some extent to classical proofs (Parigot, 1992; Barbanera and Berardi, 1996).

The Curry-Howard correspondence may also help in understanding some of the reasons for the slow recognition of a computer aided, strictly formal approach in the mathematical community and, conversely, of the moderate interest of computer scientists for its application in this field. The point is that formal proofs, whatever effort you make to write 
them in a natural, declarative style (see e.g. (Wenzel, 1999)), still look like programs, and the vast majority of mathematicians dislike programs altogether. On the other side, computer scientists (usually) like them, but all the fun is in creating something executable while, of course, you never eliminate cuts from mathematical proofs (Boolos, 1984). There is, alas, nothing so irremediably static, somberly boring as a (formal) proof of a mathematical statement, once it has been completed. Still, of course, there is the possibility to extract a program from a (constructive) proof. Unfortunately, you have no chance to extract a good algorithm from a good proof, simply because the criteria used to evaluate proofs (elegance, conciseness) and algorithms (performance, first of all) are completely different, while the proof and the program realizing it are essentially isomorphic. For instance, if you try to prove that any list of objects can be ordered with respect to a given ordering relation, you will most likely end up with a proof corresponding to an insertion algorithm; to extract a quicksort, you have to entirely rethink the proof according to the expected output (that is not methodologically very far from first writing the algorithm and then proving its correctness).

\section{Proofs and refutations}

We believe that, in the end, it is a social process that determines whether mathematicians feel confident about a theorem - and we believe that, because no comparable social process can take place among program verifiers, program verification is bound to fail.

- R.A. De Millo, R.J. Lipton, A.J. Perlis (DeMillo et al., 1979)

A theorem either can or cannot be derived from a set of axioms. I don't believe that the correctness of a theorem is to be decided by a general election.

- LeSLiE LAmport (Lamport, 1979)

There has always been an interesting debate about the actual role of proofs in mathematics. De Millo et al. firmly negate any deductive validity to proofs. This position is very common among mathematicians, and had eminent supporters. For instance G.H. Hardy, who is traditionally credited with reforming British mathematics by bringing rigor into it, described the notion of mathematical proof as we working mathematicians are familiar with in the following terms (Hardy, 1928):

There is strictly speaking no such thing as a mathematical proof; we can, in the last analysis, do nothing but point; [...] proofs are what Littlewood and I call gas, rhetorical flourishes designed to affect psychology, pictures on the board in the lecture, devices to stimulate the imagination of pupils.

This view is traditionally contrasted by the logistic (neopositivist) school, re-invigorated by the recent results in the field of automation of formal reasoning:

The history of mathematics has stories about false results that went undetected for long periods of time. However, it is generally believed that if a published mathematical argument is not valid, it will be eventually detected as such. While the process of finding a proof may require creative insight, the activity of checking a given mathematical argument is an objective activity; mathematical correctness should not be decided by a social process (Coquand, n.d.). 
Harrison explicitly mentions that one of the goals of computer aided verification should be (Harrison, 2008):

supplementing or even partly replacing the process of peer review for mainstream mathematical papers with an objective and mechanizable criterion for the correctness of proofs.

As suggested among others by MacKenzie (MacKenzie, 2005), the two positions can be reconciled if we accept the idea that proof assistants are going to change the "whole concept of proof", splitting the two roles of message and certificate:

Ever since Euclid, mathematical proofs have served a dual purpose: certifying that a statement is true, and explaining why it is true. Now those two epistemological functions may be divorced. In the future the computer assistant may take care of the certification and leave the mathematicians to look for an explanation that humans can understand.

A proof serves two purposes: (1) to be able to verify the validity of a statement and (2) to explain - by providing intuitions - why the statement is true. These roles are traditionally interwoven: a proof contains intuitions, sometimes remarks on why a certain method does not work, motivating examples, plus a line of reasoning that builds up the precise argument. With the advent of tools like proof assistants, it becomes realistic that we leave the first incarnation of a proof (the verification) to a computer and the second (the explanation) to the human.

In the optimal scenario, we can maintain a close connection between the two incarnations of proof. Or even better, we would be able to automatically generate a machine checkable certificate from a human readable message. The problem is that, since the translation has to be done automatically, the message itself must be already written in a machine understandable language, and it is extremely difficult to define a "high level" language suitable to this kind of human-machine communication.

However, there is another problem. Suppose we have Harrison's tool (and we are still very far from such a goal). Of course, the fact that proofs are validated by the tool is not a sufficient reason to accept a scientific contribution; Harrison does not suggest that, but seems to suggest the converse, that looks equally problematic.

The historical relevance of wrong proofs for the development of mathematics can be easily documented (see e.g. (Lecat, 1939)). To take a recent example well known in the programming language community - without the publication of Martin-Löf's mistaken proof of termination for system U, we would probably never have had Girard's system $\mathrm{F}$, i.e. the polymorphic lambda calculus.

False proofs, or proofs becoming refutable under suitable concept-stretching of the relevant notions are an essential component of the quasi-empirical nature of mathematics extensively discussed in Lakatos (Lakatos, 1976):

"Certainty" is far from being a sign of success, it is only a symptom of lack of imagination, of conceptual poverty. It produces smug satisfaction and prevents the growth of knowledge.

The risk envisaged by Lakatos (but similar criticism addressed to logical positivism has been raised by Popper (Popper, 1963)) is to:

construct formalized languages in which artificially congealed states of science are expressed. [...] Science teaches us not to respect any given conceptual-linguistic framework lest it should turn into a conceptual prison. 
However, Lakatos seems to be more concerned with the declarative, descriptive level of theories and definitions, than with the foundational, logical layer, and a few pages later he observes:

Nineteenth-century mathematical criticism stretched more and more concepts, and shifted the meaning-load of more and more terms onto the logical form of the propositions and onto the meaning of the few (as yet) unstretched terms. In the 1930's this process seemed to slow down and the demarcation line between unstretchable (logical) terms and stretchable (descriptive) terms seemed to become stable. A list, containing a small number of logical terms came to be widely agreed upon, so that a general definition of logical truth became possible. [...] The most interesting result in this direction was Popper (Popper, 1948) from which it follows that one cannot give up further logical constants without giving up some basic principles of rational discussion.

The only actual philosophical danger of a strictly formal approach to mathematics - not to be underestimated - may be to induce the fallacy of deductionism, suggesting that the path of discovery is from axioms and/or definitions to proofs and theorems (a risk that Lakatos considers just a bit more dangerous for mathematics, than inductivism). This point has been clearly stated by Paul Halmos in his "Automathography" (Halmos, 1985):

Mathematics is not a deductive science. When you try to [solve a problem] ... what you do is trial and error, experimentation, guesswork. You want to find out what the facts are, and what you do is in that respect similar to what a laboratory technician does, but it is different in its degree of precision and information.

A systematic use of an automatic checker to rule out wrong theorems, simply negating their right to existence and with them any form of refutation (and hence of criticism), would of course be a dramatic step towards a strictly conservative deductionist attitude, negating the possibility and importance of what Lakatos calls naive guessing and concept stretching. However, this is not its intended use. In exactly the same way as during program compilation, the process of type checking is not meant to simply discriminate good programs from bad ones. The type checker is an important driver during the program development phase, and a major tool for the deployment of lightweight, adaptive software methodologies, requiring frequent modifications and refactoring. This interactive use of the type-checker is likely to further grow in the near future; the situation is so described in (Altenkirch et al., n.d.):

Programming is a complex task which can be made easier for people to do with the help of computers. The conventional cycle of programming with a text editor then compiling in "batch mode" is a welcome shortening of the feedback loop since the days of punched cards, but it clearly under utilizes the technology available today. Any typed programming language can benefit from the capacity - but not necessarily the compulsion - to invoke the type-checker incrementally on incomplete subprograms whilst they are under development. The more powerful the type system, the more pressing this need becomes - it just gets harder to do in your head, especially when types contain computations, for which computers are inherently useful.

This interactive use of the computer is precisely the most exciting prospect in computer assisted reasoning, and the crucial point where modern interactive proof assistants differ from their first generation precursors. The goal (still extremely far, but clearly identifiable) would be to assist mathematicians not in the act of checking the "correctness" of their reasoning, but in the process of mathematical discovery, i.e. during design, analysis, 
Table 4. An extended analogy

\begin{tabular}{ll}
\hline \hline Mathematics & Programming \\
\hline $\begin{array}{l}\text { proof analysis } \\
\text { proof/thought experiment }\end{array}$ & type inference/refinement \\
untyped program \\
\hline \hline
\end{tabular}

and elaboration. For instance, in the investigation of the impact of small, local modifications in the meaning of entities on the logical correctness of the proof. This can be more easily understood by a simple extension of the Curry-Howard correspondence, by distinguishing between raw (pre-typed/untyped) programs and well-typed ones. A mathematical proof, in Lakatos' sense, is a raw program: a crystal clear thought experiment, or construction. In this perception, "proofs" prove nothing: they are just tests, experiments, not necessarily leading to the expected results $\$$. It is the process of proof-analysis that adds a deductive layer to the proof, inferring proof-generated lemmas and concepts. Proof analysis is then akin to type inference. In the realm of interactive theorem provers, the module in charge of type inference, synthesizing or constraining information omitted by the user, inserting coercions, imposing suitable views and so on, is traditionally called the refiner. The refiner (and not the kernel in charge of proof checking) is the real heart of these systems, and the primary source of their "intelligence". The constant improvements in the functionalities of this component is one of the main research trends in the field of interactive theorem proving. In particular, most of the studies aim to attain a tighter integration between the refiner and the modules in charge of proof automation, with the attempt to add limited deduction abilities to the former, along an interesting and synergistic analogy with similar studies on type systems for programming languages (see e.g. the recent, parallel investigations of type classes (Wadler and Blott, 1989; Hall, Hammond, Jones and Wadler, 1996; Wenzel, 1997; Sozeau and Oury, 2008)).

\section{Mathematics and Computer Science}

The only feasible way of coming to grips with really radical novelty is orthogonal to the common way of understanding: it consists in consciously trying not to relate the phenomenon to what is familiar from one's accidental past, but to approach it with a blank mind and to appreciate it for its internal structure.

- EdsGer Dijkstra (Edsger.W.Dijkstra, 1986)

As observed by Van Den Bos (Bos, 1979), the real novelty of De Millo et al. paper was that

for the first time a paper on the philosophy of computer science, in this case the methodology of program verification, has been published in Communications of ACM.

I As Lakatos says, after Columbus, one should not be surprised if one does not solve the problem one has set out to solve. 
Here, De Millo, Lipton and Perlis seem to have lost a great opportunity, failing to exploit the most interesting aspects of the analogy they had put forth, entrenching themselves behind a strictly sociological position and making use of old slogans like "dullness of rigor", "artificiality versus beauty", and similar things.

Actually, there are at least two major novelties introduced by the advent of computer science in the epistemological debate: the first one is related to the intrinsic nature of computer science, that strongly differentiates it from mathematics (and all other scientific disciplines), while the second one concerns the altered conditions induced by an extensive use of information technology in scientific practice.

Concerning the first point, if we look back at the Curry-Howard-De Millo correspondence, there is a striking difference between mathematics and computer science that should be evident at first glance. The point is that while programs (not algorithms, but programs!) are a major object of study, analysis and elaboration for computer science, the mathematical investigation of proofs is absolutely marginal, essentially confined to a minor subfield of logic known as "proof theory", see e.g. (Prawitz, 1965; Girard, Lafont and Taylor, 1989).

This is not surprising since computer science is about information, its automatic processing, transformation, and communication, and the main vehicle for managing information are programs (again, programs, not algorithms!). Luckily, programs are informative entities, and not only can they be the object of a metamathematical investigation, but can be also processed, transformed and communicated as any other kind of information. In fact, computer science starts to become really interesting when it is applied to itself. It is precisely this circularity, this auto-referentiality of computer science that makes it entirely peculiar among all scientific disciplines: meta-information is still another form of information; the techniques and methodologies of computer science are an essential part of its domain of investigation.

The difference with mathematics is striking, since the mathematical method has never been the object of a serious mathematical investigation (if not, possibly, for the timid, limited attempts of neopositivism). We should then acknowledge, following Popper, that since it cannot be made an object of validation experiments, and cannot be possibly refuted, the celebrated "mathematical rigor" is a purely ideological claim, a mere illusion, or if you prefer the result of a refined liturgy.

A program is written in a strictly formal language, and the possibility of writing long programs testifies by itself the possibility of writing long formal proofs. Moreover, since programs (almost always) work, there must be a way to govern the pedantic complexity of formal languages. Here, we are completely reversing the traditional position: the point is not that verification is important because programs crash, but that verification must be possible because, most of the time, they don't crash.

An external observer might believe that this is due to particularly favorable, peculiar conditions of computer science, but this is not the case. The multilingual foundational miasma is a reality we have learned to live with (and, to some extent, to appreciate) at the descriptive/functional level. Change is a rule and adaptability a bare necessity (Fowler, n.d.): 
There's a refrain I've heard on every problem project I've run into. The developers come to me and say "the problem with this project is that the requirements are always changing". The thing I find surprising about this situation is that anyone is surprised by it. In building business software requirements changes are the norm, the question is what we do about it.

Hence, admitting that long, sensible formal programs/proofs can be written, the really interesting question is if some of the methodologies, not only of static analysis but more generally of software development, can be applied to the realm of mathematics. In this way, the original verification/type checking problem is put in its correct perspective, namely as one of the tools contributing to a comfortable development environment for the "working mathematician".

Here we also directly arrive at our second point, namely the exploitation of the potentialities offered by the new information technologies, and their impact on scientific practice. According to Popper, scientific rigor does not depend on the objectivity or critical attitude of individuals, but on the methodology employed (here, Kuhn expresses similar ideas (Hutcheon, 1995), but in terms of standards and values of science dictated by the "paradigm" adopted by the scientific community). Hence, if the advent of technology will sensibly affect the methodology, the notion of rigor will change accordingly, and with it the essence of the entire scientific discipline. The point is particularly important in the case of mathematics, where changes in the criterion of "rigor of the proof" engender major revolutions (Lakatos, 1976).

By many symptoms, we are approaching one of Kuhn's pre-revolutionary crisis in the realm of mathematics. The big novelty of this crisis is due to the introduction of the use of the computer to master the growing complexity of mathematical proofs. Standard methods seem to have hit a ceiling, not for intrinsic deficiencies of the theoretical framework, but for a human deficiency to cope with complex computations/encodings. As observed by Sarnak (Economist, n.d.), one of the editors of the Annals of Mathematics, they expect to receive a growing number of papers involving computer code in the next 20-50 years. Then, mathematics may become a bit like experimental physics - as foreseen by Sarnak - where certain results are taken on trust, and independent duplication of experiments replaces examination - or, as attempted by Hales in his Flyspeck project, we may try to use the computer as a remedy as well. In both cases the notion of "mathematical rigor" will be deeply affected.

\section{Content and semantics}

One of the major goals of verification is to provide a new dimension in the way we do mathematics, as well as in the way we do computer science.

- W.D. Maurer (Maurer, 1979)

The idea that a proof assistant should not just support the process of mathematical verification, but that of mathematical discovery was already clearly outlined by Constable et al. (Constable et al., 1986) in their description of the Nuprl system:

For our intention is to provide a medium for doing mathematics different from that provided by paper and blackboard. Eventually such a medium may support a variety of input devices and 
may provide communication with other users and systems; the essential point, however, is that this new medium is active, whereas paper, for example, is not.

In the nineties this goal was somehow blurred by the imposing pronouncement of the QED manifestoll that, with its taxing goal to provide a cultural monument to "the fundamental reality of truth" shifted back the focus on formal verification.

$Q E D$ is the very tentative title of a project to build a computer system that effectively represents all important mathematical knowledge and techniques. The QED system will conform to the highest standards of mathematical rigor, including the use of strict formality in the internal representation of knowledge and the use of mechanical methods to check proofs of the correctness of all entries in the system.

The manifesto describes the ambitious goals of the project and discusses questions and doubts and the answers to them. In 1994 and 1995 there have been two workshops on QED, but after that no more. Is the QED manifesto too ambitious? In this respect it is instructive to read what the authors of the QED manifesto thought was needed to be done. First of all, a group of enthusiastic scientists should get together to determine which parts of mathematics are needed to be formalized, in what order and with which cross-connections. The authors assume that this phase may take a few years and it may even involve a rearrangement of the mathematics itself, before the actual formalization work can start. Other points in this 'to-do-list' are of a similar top-down nature.

However, this is a rather old fashioned approach to the problems, focusing solely on formal correctness. Developments like Wikipedia show that a more 'bottom up' distributed approach may work better, using a simple lightweight basic technology. One could claim that for mathematics - where the end goal is to get a library of verified reliable results - such an approach could never work, but for Wikipedia the same doubts were raised at first: Wikipedia is typically something that works in practice but not in theory. (See (Wiedijk, 2007) for a present day evaluation of the QED manifesto.

The goal of developing innovative, semantic based functionalities transcending the mere operation of formal checking, and focusing on problems related to the management of the repository of (formal) mathematical theorems, such as archiving, indexing, searching, communication and publishing was strongly advocated in (Asperti, Padovani, Sacerdoti Coen and Schena, 2000). The emerging XML technology seemed to provide the natural infrastructure for the development of the new systems. In particular, the idea was to use XML as a main, platform independent language for long-term representation and exchange of the naturally structured, formal mathematical knowledge, exploiting to their full extent all kinds of XML technologies: MathML and XHTML for rendering; XSLT for the application of notational transformations; Xpath and XQuery for complex, content based queries; and RDF for indexing and efficient document retrieval. It is a pity that, since then, most of the expectations about XML technologies have not been fulfilled due to intrinsic deficiencies in their design and implementation: MathML failed to be adopted by major browsers; XSLT is just too prolix for simple operations and too weak for more

\| http://www-unix.mcs.anl.gov/qed 
complex "content sensitive" operations; XQuery is too slow for large, highly structured data bases; and RDF never really went beyond the project phase.

An alternative attempt to employ XML for encoding mathematical content was made by the OpenMath project ((ed.), 2000). In Dewar's words, OpenMath is a standard for representing mathematical data in as unambiguous a way as possible. Essentially, an OpenMath object is a labeled tree describing the abstract syntax tree of the mathematical entity, whose leaves are the basic OpenMath data structures, such as IEEE double precision floats; Unicode strings; byte arrays, variables or symbols. Symbols consist of a name and a reference to a "definition" in an external document called a content dictionary (CD). The definition itself is given in natural language, while CD's are essentially meant as background references for the implementers of phrasebooks, i.e. of the actual software tools able to internalize the OpenMath object inside specific applications.

A ferocious critique of OpenMath is contained in (Fateman, n.d.). Although we share most of Fateman's thoughts, and in particular the concern about the lack of any serious proof of concept, there are a few points that probably deserve a deeper discussion. In particular, Fateman affirms that

All protestations to the contrary, it [OpenMath] simply does not have any mandate outside the rather simple application of denoting what could be trivially done in any programming language capable of representing attributed trees.

This is true, but the point is not to just use abstract syntax trees for representing the information, but to agree on their syntax, i.e. to propose a standard. Sharing a common grammar seems to be a minimal pre-requisite for any possible kind of communication between automatic devices. Then, the use of XML is indeed not essential, but quite natural. The tremendous step forward consisting in passing from an unstructured representation of the information to a structured (standard) one, simply cannot be ignored. Of course, what makes a standard is not a self-proclamation, but its actual adoption, and OpenMath clearly failed in its mission. However, this does not imply that the objective was basically wrong.

The second point is more delicate. Fateman says:

We learn that each corresponding program X must have a phrasebook which converts its internal form $Y$ to an OpenMath form which is, one hopes, the universal semantic notion of $Y$. But it seems that except in trivial matters, its semantics may have to be encoded as "the meaning of $Y$ to the program X". Thus the ideal of having $n$ programs communicating using $n$ phrasebooks to/from OpenMath has been lost.

Of course, it is not the ' $n^{2}$ versus $n$ ' point that matters, but the fact that we would entirely loose the real sense of having an intermediate structure. If we cannot give any intended semantic interpretation to our syntax, what is the actual point in having it? The critique seems to irreparably undermine at its very roots the quest for a universal "intermediate" language.

In fact, on the contrary, it doesn't. Suppose we ask an application to compute a solution for a given problem $P$. We have no way to be sure that it really understood our problem. After a given time it returns a solution ' $a$ '. We have no way to be sure we correctly interpreted the solution, either. But who cares? We check if our interpretation of $a$ is a solution to our interpretation of $P$, and if it is, we are happy. The point is that the 
intermediate information is merely a witness, a trace that we have to interpret and check after internalization. The interest is, as usual, that checking is enormously less expensive than finding. The general picture is even more clear when we add proofs. Suppose, there is in our "universal" language a proof $p$ of some property $A$. We may define translations $p_{x}$ and $A_{x}$ to our internal language and check if indeed $p_{x}$ is (under our interpretation) a proof of $A_{x}$. If it is, we have a proof, without caring about the fact whether the translation was "correct" (and surely it couldn't be, since we have no semantics for the intermediate language).

Having understood that we may have an interesting intermediate language with no semantics, the actual points are:

1 Can we define a "trace" language for proofs that are of suitable interest to multiple applications?

2 Can we apply static analysis techniques for this language (e.g. a weak, possibly logically inconsistent type system);

3 Can we promote a direct collaborative development of this layer?

Regarding the first point, as we already observed, the process of formalization of a mathematical statement is often compared to the translation of a piece of code from a high-level programming language to some assembly language, corresponding to a foundational dialect in some logical system. Inter-operability at a foundational level is as hopeless as trying to send instructions from one microprocessor to another. On the other side, the most expensive part of the formalization process consists in a preliminary conceptual phase of transformation of the proof into a form suitable to be understood by a machine that is largely independent from the specific idiosyncrasies of each particular foundation. Compilation is not an atomic process, and what is currently clearly lacking is a good intermediate language - that is precisely the trace language we are talking about.

Is such an objective feasible? If we overcome the foundational impasse of fixing a formal semantics to quantifiers, and the diatribe about the role and nature of functions, it is usually acknowledged that we could probably agree on a common syntax for mathematical formulae. A minimal trace language, extremely poor but already not deprived of interest, would consist of a graph of dependencies relating a result to the main auxiliary facts required for its proof (possibly admitting multiple paths). The interest of such an approach is that it can be refined to a more or less arbitrary degree of detail, to the point where a software system can automatically fill in the missing steps. Moreover, the system itself could assist the user in this refinement activity. The other point is that the approach does not vastly differ from the natural top-down methodology already in use in wiki-like systems (where you typically first create the link and then the page you are linking). If we merely ask a typical mathematical user of such a system to type mathematical statements using a suitable set of content-oriented $\mathrm{A}_{\mathrm{A}} \mathrm{T} \mathrm{E}$-like macros (possibly to be agreed according to the same policies that usually govern these systems) we really see no reason why he should not consent (especially since he could also have some presentational benefits). Quoting Hales again (Hales, 2008):

To undertake the formalization of just 100,000 pages of core mathematics would be one of the most ambitious collaborative projects ever undertaken in pure mathematics, the sequencing of a 
mathematical genome. One might imagine a massive wiki collaboration that settles the text of the most significant theorems in contemporary mathematics from Poincaré to Sato-Tate.

The point is that everybody must be able to contribute, independent of the fact whether or not using a proof assistant. Interactive theorem-prover users can be in charge of refining the proofs to more elementary components, possibly automatically populating the library of basic results (mostly meant to be only inspected by automatic devices). At the same time, the refinement process can provide an essential feedback to higher-levels, possibly requiring sensible revisiting of already codified notions and results.

Preliminary developments towards a "MathWiki" system that supports the distributed development of a library of mathematics on various levels of formality, ranging from the mathematics informally described on existing web pages to the formalized mathematics that we encounter in proof assistants, have been described and advocated in (Corbineau and Kaliszyk, 2007; Corbineau, Geuvers, Kaliszyk, McKinna and Wiedijk, 2008). Such a system should provide a lightweight cooperative framework for developing and discussing mathematics, on various levels of formality. Also it should provide a place where various existing repositories of (formal) mathematics come together.

\section{Conclusion}

The lack at this late date of even a single verification of a working system has sometimes been attributed to the youth of the field. ... there has never been a verification of, say, a Cobol system that prints real checks; lacking even one makes it seem doubtful that there could at some time in the future be many.

- R.A. De Millo, R.J. Lipton, A.J. Perlis (DeMillo et al., 1979)

As we already observed, the gloomy predictions of De Millo et al. have been largely refuted. Formal verification is at present a concrete reality, permitting correctness proofs of complex software applications. For instance, in the framework of the Verifix Project a compiler from a subset of Common Lisp to Transputer code was formally checked in PVS (see (Dold and Vialard, 2001)). Strecker (Strecker, 1998) and Klein (Klein, 2005) certified bytecode compilers from a subset of Java to a subset of the Java Virtual Machine in Isabelle. In the same system, Leinenbach (Leinenbach, Paul and Petrova, 2005) formally verified a compiler from a subset of C to a DLX assembly code. The Compcert project, headed by Xavier Leroy, has recently produced a verified optimizing compiler from $\mathrm{C}$ to PowerPC assembly code, based on the use of the Coq proof assistant both for programming the compiler and proving its correctness (Leroy, 2006; Tristan and Leroy, 2008). Similar achievements have been obtained in other fields of computer science, spanning from hardware (Harrison, 2007) to operating systems (?; Klein, 2009).

The parallel made by De Millo, Lipton and Perlis between computer science and mathematics is however still relevant, and possibly even more actual in view of the recent proliferation of mathematical proofs involving the use of computers. In particular, in this paper we argued that mathematics is destined to assimilate some practices of software development, and that the future of both mathematics and programming lies in the 
fruitful combination of formal verification and the usual social processes that are already working in both scientific disciplines. Quoting Hales (Hales, 2008):

The hope is the system [the proof assistant] will eventually become sufficiently user-friendly to become a familiar part of the mathematical workplace, much as email, $T_{E} X$, computer algebra systems and Web browsers are today.

At present, we are still very far from this dream; the current cost to transcribe a printed page of textbook mathematics into machine-checkable code is estimated as a week's labor in (Hales, 2008), and, more pessimistically, as 1.5 hours per line in (Asperti and Ricciotti, 2009). In (Wiedijk, n.d.) the cost of formalizing the standard bachelor's curriculum of mathematics is estimated at 140 man years. The point is not only to reduce this cost, but also to improve the benefits coming from the representation of the information in a "machine understandable" richly structured format, suitable to be elaborated by a machine. This means developing innovative, content-based functionalities, eventually overcoming the reductive operational perspective of verification. The research directions were clearly traced by Constable et. al (Constable et al., 1986) more than 20 years ago:

The natural growth path for a system like Nuprl tends toward increased "intelligence". [...] For example, it is helpful if the system is aware of what is in the library and what users are doing with it. It is good if the user knows when to involve certain tactics, but once we see a pattern to this activity, it is easy and natural to inform the system about it. Hence there is an impetus to give the system more knowledge about itself.

Unfortunately, the progress in this direction is extremely slow, and the following question of Dijkstra remains, for the moment, still unanswered:

In the relation between mathematics and computing science, the latter has been for many years at the receiving end, and I have often asked myself if, when, and how computing would ever be able to repay its debt.

- Edsger Dijkstra (Edsger.W.Dijkstra, 1986)

\section{References}

Alkassar, E., Bogan, S. and Paul, W. J. (2009). Proving the correctness of client/server software, Sadhana 34(1): 145-191.

Altenkirch, T., McBride, C. and McKinna, J. (n.d.). Why Dependent Types Matter http://sneezy.cs.nott.ac.uk/epigram/.

Asperti, A., Padovani, L., Sacerdoti Coen, C. and Schena, I. (2000). Content-centric logical envirnoments, Short Presentation at the Fifteenth IEEE Symposium on Logic in Computer Science.

Asperti, A. and Ricciotti, W. (2009). About the formalization of some results by Chebyshev in number theory, Proceedings of TYPES'08, Vol. 5497 of LNCS, Springer-Verlag, pp. 19-31.

Avigad, J., Donnelly, K., Gray, D. and Raff, P. (2007). A formally verified proof of the prime number theorem, ACM Trans. Comput. Log. 9(1).

Barbanera, F. and Berardi, S. (1996). A Symmetric Lambda Calculus for Classical Program Extraction, Inf. Comput. 125(2): 103-117.

Boolos, G. (1984). Don't eliminate cut, Journal of Philosophical Logic 13: 373-378.

Bos, J. V. D. (1979). Letter to the editor, Communications of the ACM 22: 623. 
Bourbaki, N. (1968). Theory of Sets, Elements of mathematics, Addison Wesley.

Constable, R. L., Allen, S. F., Bromley, H. M., Cleaveland, W. R., Cremer, J. F., Harper, R. W., Howe, D. J., Knoblock, T. B., Mendler, N. P., Panangaden, P., Sasaki, J. T. and Smith, S. F. (1986). Implementing Mathematics with the Nuprl Development System, Prentice-Hall, NJ. Coquand, T. (n.d.). Draft of the Formath Project.

Corbineau, P., Geuvers, H., Kaliszyk, C., McKinna, J. and Wiedijk, F. (2008). A real semantic web for mathematics deserves a real semantics., in C. Lange, S. Schaffert, H. Skaf-Molli and M. Völkel (eds), SemWiki, Vol. 360 of CEUR Workshop Proceedings, CEUR-WS.org.

URL: http://sunsite.informatik.rwth-aachen.de/Publications/CEUR-WS/

Corbineau, P. and Kaliszyk, C. (2007). Cooperative repositories for formal proofs - a wiki-based solution, in M. Kauers, M. Kerber, R. Miner and W. Windsteiger (eds), Towards Mechanized Mathematical Assistants, Vol. 4573 of LNCS, Springer, pp. 221-234.

DeMillo, R. A., Lipton, R. J. and Perlis, A. J. (1979). Social Processes and Proofs of Theorems and Programs, Commun. ACM 22(5): 271-280.

Dold, A. and Vialard, V. (2001). A Mechanically Verified Compiling Specification for a Lisp Compiler, in R.Hariharan, M.Mukund and V. Vinay (eds), Proc. of the 21st Conference on Foundations of Software Technology and Theoretical Computer Science (FSTTCS 2001), Bangalore, India., Vol. 2245 of LNCS, Springer, pp. 144-155.

Economist, T. (n.d.). Proof and Beauty, march 31st 2005.

(ed.), M. D. (2000). Special issue on OpenMath, ACM SIGSAM Bulletin 34.

Edsger.W.Dijkstra (1986). On a cultural gap, Mathematical Intelligencer 8(1): 48-52.

Fateman, R. (n.d.). A Critique of OpenMath and Thoughts on Encoding Mathematics, http://www.eecs.berkeley.edu/ fateman/papers/openmathcrit.pdf.

Feit, W. and Thompson, J. G. (1963). Solvability of groups of odd order, Pacific Journal of Mathematics 13: 775-1029.

Fowler, M. (n.d.). The New Methodology http://www.martinfowler.com/articles/newMethodology.html.

Geuvers, H. (2009). Proof Assistants: history, ideas and future, Sadhana 34(1): 3-25.

Girard, J.-Y., Lafont, Y. and Taylor, P. (1989). Proofs and Types, Cambridge Tracts in Theoretical Computer Science, Cambridge University Press.

Gonthier, G. (2008). Formal Proof - The four color theorem, Notices of the American Mathematical Society 55: 1382-1394.

Gonthier, G. (December 15-17, 2007). The four colour theorem: Engineering of a formal proof, Proceedings of the 8th Asian Symposium on Computer Mathematics, ASCM 200\%, Singapore, p. 333 .

Gonthier, G., Mahboubi, A., Rideau, L., Tassi, E. and Thery, L. (2007). A modular formalisation of finite group theory, The 20th International Conference on Theorem Proving in Higher Order Logics, Vol. 4732, pp. 86-101.

Hales, T. (2008). Formal Proof, Notices of the American Mathematical Society 55: 1370-1381.

Hales, T. C. (2005). A proof of the kepler conjecture, Ann. Math. 162: 1065-1185.

Hales, T. C. (2007). The jordan curve theorem, formally and informally, The American Mathematical Monthly 114: 882-894.

Hall, C., Hammond, K., Jones, S. P. and Wadler, P. (1996). Type classes in haskell, ACM Transactions on Programming Languages and Systems 18: 241-256.

Halmos, P. (1985). I want to be a Mathematician: An Automathography.

Hamid, N. A., Shao, Z., Trifonov, V., Monnier, S. and Ni, Z. (2003). A Syntactic Approach to Foundational Proof-Carrying Code, J. Autom. Reasoning 31(3-4): 191-229.

Hardy, G. H. (1928). Mathematical proof, Mind 38: 1-25. 
Harrison, J. (2007). Floating-Point Verification, J. UCS 13(5): 629-638.

Harrison, J. (2008). Formal Proof - Theory and Practice, Notices of the American Mathematical Society 55: 1395-1406.

Hoare, C. A. R. (1969). An Axiomatic Basis for Computer Programming, Commun. ACM 12(10): 576-580.

Howard, W. A. (1980). The formulae-as-types notion of construction, in J.P.Seldin and J.R.Hindley (eds), To H.B. Curry: Essays on Combinatory Logic, Lambda Calculus and Formalism, Academic Press, Boston MA, pp. 479-490.

Hutcheon, P. D. (1995). Popper and Kuhn on the Evolution of Science, Brock Review 4(1/2): 2837.

Klein, G. (2005). Verified Java Bytecode Verification, Information Technology 47(2): 107-110.

Klein, G. (2009). Operating system verification - an overview, Sadhana 34(1): 27-69.

Lakatos, I. (1976). Proofs and Refutations: The Logic of Mathematical Discovery, Cambridge University Press.

Lamport, L. (1979). Letter to the editor, Communications of the ACM 22: 624 .

Langley, S. P. (1891). Experiments in aerodynamics, Kessinger Publishing.

Lecat, M. (1939). Erreurs de mathématiciens: des origines à nos jours., Ancienne Librairie Castaigne, Brussels.

Lee, J. K. (2002). Philosophical perspectives on proof in mathematics education, Philosophy of Mathematics Education Journal 16.

Leinenbach, D., Paul, W. J. and Petrova, E. (2005). Towards the Formal Verification of a C0 Compiler: Code generation and implementation correctnes, Third IEEE International Conference on Software Engineering and Formal Methods (SEFM 2005), 7-9 September 2005, Koblenz, Germany, IEEE Computer Society, pp. 2-12.

Leroy, X. (2006). Formal certification of a compiler back-end or: programming a compiler with a proof assistant, Proceedings of the 33rd ACM SIGPLAN-SIGACT Symposium on Principles of Programming Languages, POPL 2006, Charleston, South Carolina, USA, pp. 42-54.

MacKenzie, D. (2005). What in the name of Euclid is going on here?, Science 207(5714): 14021403.

Maurer, W. D. (1979). Letter to the editor, Communications of the ACM 22: 625-629.

Necula, G. C. and Lee, P. (1996). Proof-Carrying Code, Technical Report CMU-CS-96-165, Carnegie Mellon University.

Parigot, M. (1992). Lambda-Mu-Calculus: An Algorithmic Interpretation of Classical Natural Deduction, Proc. of the Logic Programming and Automated Reasoning International Conference LPAR'92, St. Petersburg, Russia, July 15-20, 1992, Vol. 624 of LNCS, Springer, pp. 190201.

Popper, K. (1948). Logic without assumptions, Aristotelian society proceedings 47: 251-292.

Popper, K. (1963). Conjectures and Refutations. The Growth of Scientific Knowledge, Routledge. Prawitz, D. (1965). Natural Deduction: a proof theoretical study, Almqvist \& Wiksell.

Schieber, P. (March/April 1987). The Wit and Wisdom of Grace Hopper, OCLC Newsletter 167.

Sozeau, M. and Oury, N. (2008). First-class type classes, TPHOLs, pp. 278-293.

Strecker, M. (1998). Construction and Deduction in Type Theories, PhD thesis, Universität Ulm.

Tristan, J.-B. and Leroy, X. (2008). Formal verification of translation validators: a case study on instruction scheduling optimizations, Proceedings of the 35th ACM SIGPLAN-SIGACT Symposium on Principles of Programming Languages, POPL 2008, San Francisco, California, pp. $17-27$. 
Wadler, P. and Blott, S. (1989). How to make ad-hoc polymorphism less ad hoc, POPL '89: Proceedings of the 16th ACM SIGPLAN-SIGACT symposium on Principles of programming languages, ACM, New York, NY, USA, pp. 60-76.

Wenzel, M. (1997). Type classes and overloading in higher-order logic, TPHOLs, pp. 307-322.

Wenzel, M. (1999). Isar - a generic interpretative approach to readable formal proof documents, Theorem Proving in Higher Order Logics, Vol. 1690 of LNCS, Springer, pp. 167-184.

Wiedijk, F. (2007). The qed manifesto revisited, in R. Matuszwski and A. Zalewska (eds), From Insight to Proof, Festschrift in Honour of Andrzej Trybulec, Vol. 10(23) of Studies in Logic, Grammar and Rhetoric, University of Białystok, pp. 121-133.

Wiedijk, F. (n.d.). Estimating the cost of a standard library for a mathematical proof checker , http://www.cs.ru.nl/ freek/notes/mathstdlib2.pdf. 